\title{
DESENVOLVIMENTO INICIAL E EFICIÊNCIA DE USO DE ÁGUA E NITROGÊNIO POR MUDAS DE Calophyllum brasiliense, Eucalyptus urograndis, Tabebuia impetiginosa $\mathbf{E}$ Toona ciliata ${ }^{l}$
}

\author{
EARLY DEVELOPMENT AND EFFICIENCY IN WATER AND NITROGEN USE BY SEEDLINGS \\ OF Calophyllum brasiliense, Eucalyptus urograndis, Tabebuia impetiginosa AND Toona ciliata
}

Anderson Fernandes Souza ${ }^{1}$ Elio de Oliveira Rocha Junior ${ }^{2}$ Valdemir Antônio Laura ${ }^{3}$

\section{RESUMO}

O conhecimento sobre o desenvolvimento inicial das mudas é fundamental para o sucesso de atividades de reflorestamento e de plantio em florestas naturais. O objetivo deste trabalho foi avaliar o desenvolvimento inicial e a eficiência de uso de água e nitrogênio por mudas de quatro espécies florestais nativas e exóticas (Calophyllum brasiliense (nativa), Eucalyptus urograndis (exótica), Toona ciliata (exótica) e Tabebuia impetiginosa (nativa)) com idade de aproximadamente 120 dias após a semeadura por 120 dias após o transplante em condições de casa de vegetação. Foram realizadas amostragens destrutivas para avaliar a biomassa seca (folha, caule, raiz e total), área foliar, relação raiz/parte aérea e nitrogênio total nas mudas. Com base nessas variáveis, foram calculados os parâmetros fisiológicos de crescimento e a eficiência de uso de água, que foi obtida pela razão entre o total de biomassa seca acumulada e a evapotranspiração dos vasos no período, determinada através de pesagens diárias em balança de bancada. Ao final de 240 dias após a semeadura, Calophyllum brasiliense e Tabebuia impetiginosa apresentaram maior produção de biomassa seca total e Toona ciliata apresentou maior relação raiz/parte aérea. Não houve diferenças entre a taxa de crescimento relativo das espécies avaliadas. Eucalyptus urograndis, Toona ciliata e Calophyllum brasiliense apresentaram os maiores valores de razão de área foliar, Toona ciliata, Tabebuia impetiginosa e Eucalyptus urograndis apresentaram os maiores valores de área foliar específica, Eucalyptus urograndis e Calophyllum brasiliense apresentam os maiores valores para razão de massa foliar e Tabebuia impetiginosa, Toona ciliata e Eucalyptus urograndis apresentaram os maiores valores de taxa de assimilação líquida. Com base na quantificação do nitrogênio total, Tabebuia impetiginosa e Toona ciliata foram as espécies mais eficientes no uso do nitrogênio. Eucalyptus urograndis apresentou a maior evapotranspiração enquanto Calophyllum brasiliense e Tabebuia impetiginosa foram as espécies mais eficientes no uso de água.

Palavras-chave: análise de crescimento; consumo de água; espécies florestais; espécies nativas.

\section{ABSTRACT}

The knowledge about the early development of seedlings is critical to the success of reforestation and planting in natural forests. The aim of this study was to evaluate the initial development and efficiency of water and nitrogen use by seedlings of four native and exotic species (Calophyllum brasiliense (native), Eucalyptus urograndis (exotic), Toona ciliata (exotic) and Tabebuia impetiginosa (native)) with age of approximately 120 days after sowing for 120 days after transplantation in green-house conditions. Destructive samples were carried out to assess the dry biomass (Leaf, Stem, Root and Total), leaf area, the root shoot ratio and total nitrogen in seedlings. Based on these variables, it was calculated the physiological parameters of growth and efficiency of water use, which was obtained by the ratio between the total dry biomass

1 Biólogo, Dr., Professor do CEFET-RJ, Unidade Descentralizada de Nova Friburgo, Centro Federal de Educação Tecnológica Celso Suckow da Fonseca, Av. Governador Roberto Silveira, 1900, CEP 28635-000, Nova Friburgo (RJ), Brasil. anderson.souza@cefet-rj.br

2 Biólogo, MSc., Pós-graduação em Biologia Vegetal, Instituto de Biociências, Universidade Federal de Mato Grosso do Sul, Av. Costa e Silva, s/n, CEP 79070-900, Caixa Postal 549, Campo Grande (MS), Brasil. elioroch@ gmail.com

3 Agrônomo, Dr., Professor e Pesquisador da Embrapa, Embrapa Gado de Corte, Rodovia BR 262 km 4, CEP 79002-970, Caixa Postal 154, Campo Grande (MS), Brasil. valdemir@cnpgc.embrapa.br

Recebido para publicação em 11/04/2013 e aceito em 25/09/2017

Ci. Fl., v. 28, n. 4, out. - dez., 2018 
accumulation and evapotranspiration of vessels in the period, determined by daily weighing in a balance bench. At the end of 240 days after sowing, Calophyllum brasiliense and Tabebuia impetiginosa produced more total biomass and Toona ciliata showed higher root shoot ratio. There were no differences between the relative growth rate of the species evaluated. Eucalyptus urograndis, Toona ciliata and Calophyllum brasiliense showed the highest values of leaf area ratio, Toona ciliata, Tabebuia impetiginosa and Eucalyptus urograndis showed the highest values of specific leaf area, Eucalyptus urograndis and Calophyllum brasiliense show the highest values for leaf mass ratio and Tabebuia impetiginosa, Toona ciliata and Eucalyptus urograndis showed the highest values of net assimilation rate. Based on quantification of total nitrogen, Tabebuia impetiginosa and Toona ciliata were the most efficient species in the use of nitrogen. Eucalyptus urograndis presented the highest evapotranspiration while Calophyllum brasiliense and Tabebuia impetiginosa were the most efficient species in water use.

Keywords: growth analysis; water consumption; forest species; native species.

\section{INTRODUÇÃO}

A crescente demanda por madeira e outros produtos de origem florestal levou ao crescimento das áreas reflorestadas no Brasil com espécies de rápido crescimento, principalmente exóticas, ocupando aproximadamente, 5,98 milhões de ha, sendo 3,75 milhões com eucalipto, 1,8 milhões com pinus e 425,2 mil ha com outras espécies, como acácia-negra, gmelina, pópulus, seringueira, teca e araucária (SOCIEDADE BRASILEIRA DE SILVICULTURA, 2008), com destaque para a região do Cerrado, devido principalmente às condições edafoclimáticas e fisiográficas da região serem favoráveis ao estabelecimento de plantios de eucalipto (OLIVEIRA et al., 1998).

Para que o setor florestal continue seu processo de expansão, é fundamental a ampliação das pesquisas científicas e avanços técnicos visando às melhorias na produção, qualidade e diversificação das mudas, em busca de boa adaptação e bom crescimento após o plantio (GONÇALVES et al., 2000). A qualidade das mudas tem função estratégica na sobrevivência das plantas (FINGER et al., 2003).

O conhecimento sobre o desenvolvimento inicial das mudas é fundamental para o sucesso da atividade de produção de mudas de qualidade, que por sua vez é de suma importância para o sucesso de atividades de reflorestamento e de plantio em florestas naturais (MOTA; SCALON; HEINZ, 2012). Uma das ferramentas para ajudar a compreender as relações ecológicas entre o desenvolvimento inicial das mudas e o ambiente é a análise de crescimento. A análise de crescimento pode ser usada para a avaliação da produtividade de culturas e permite que se investigue a adaptação ecológica dessas culturas a novos ambientes, a competição entre espécies, os efeitos de manejo e tratamentos culturais, e a identificação da capacidade produtiva de diferentes genótipos (ALVAREZ et al., 2005). A análise quantitativa de crescimento permite a obtenção de vários índices fisiológicos, tais como a taxa de crescimento relativo, razão de área foliar e taxa de assimilação líquida, importantes para o entendimento do desenvolvimento inicial de espécies vegetais (BENINCASA, 2003). A taxa de crescimento relativo, por exemplo, é um índice de eficiência que representa a capacidade da planta em produzir material novo (AGUIAR NETTO; RODRIGUES; PINHO, 2000) e pode ser decomposta pela multiplicação entre a razão de área foliar e a taxa de assimilação líquida, na qual é possível determinar qual dessas variáveis fisiológicas contribuiu mais fortemente no crescimento relativo de cada espécie avaliada (CRISÓSTOMO; FREITAS; RODRÍGUEZ-ECHEVERRÍA, 2007).

A razão de área foliar representa a razão entre a área foliar e a biomassa seca total e é considerada como uma medida da capacidade fotossintética de uma planta, sendo esta diretamente influenciada pelas variáveis fisiológicas área foliar específica e razão de massa foliar. A área foliar específica consiste na relação entre a área foliar e a biomassa seca das folhas. É uma medida da expansão média da folha, em área, por unidade de biomassa seca foliar e define indiretamente a densidade ou espessura das folhas, estando relacionada a atributos anatômicos e estruturais das folhas (GROOM; LAMONT, 1999). Já a razão de massa foliar consiste na relação entre a biomassa seca das folhas e/ou folíolos e a biomassa total da planta, representando a porcentagem de tecido assimilatório na biomassa total (SILVA; BELTRÃO; AMORIM NETO, 2000).

Nos florestamentos há a predominância da utilização de espécies exóticas principalmente do gênero Eucalyptus sp., que dispõe de vasto material bibliográfico (ODA; MENCK; VENCOVSKY, 1989; FARIA 
et al., 2008). Contudo, existe uma tendência para a utilização de espécies nativas, seja em reflorestamentos ou plantios naturais.

Embora alguns ensaios em campo sobre o desenvolvimento de várias espécies arbóreas nativas sejam relatados (POORTER, 1999; CRUZ et al., 2004; GRAVE et al., 2007; MARIMON et al., 2008; MOTA; SCALON; HEINZ, 2012), ainda é pequena a quantidade de informações sobre o assunto, principalmente na comparação entre espécies e suas utilizações na exploração comercial. Esta constatação aponta para um grande campo potencial de pesquisa na recuperação de áreas degradadas e nos sistemas de produção, visando à obtenção de benefícios múltiplos tanto para o ambiente local quanto para seus habitantes.

Eucalyptus urograndis (Eucalyptus urophylla S. T. Blake X Eucalyptus grandis W. Hill ex Maiden.), Tabebuia impetiginosa (Mart.) Standl(Ipê roxo), Calophyllum brasiliense Camb.(Guanandi) e Toona ciliata M. Roem. (cedro-australiano) são espécies florestais de ampla utilização e/ou amplo potencial de exploração comercial em plantios puros ou em alternativas sustentáveis como os sistemas agroflorestais, sendo as duas últimas tratadas como substitutas do mogno (CARVALHO, 1994; LORENZI et al., 2003).

Em decorrência da crescente preocupação com a disponibilidade dos recursos naturais voltados para a agricultura, em especial os hídricos e nutricionais, há um interesse renovado na tentativa de desenvolver estratégias que possibilitem a utilização mais eficiente dos recursos naturais nos sistemas de cultivo através de melhores práticas, por exemplo, com a seleção de variedades tolerantes à seca, ou na seleção de espécies mais adequadas a cada região (DOORENBOS; KASSAN, 1979; HATIFIELD; SAUER; PRUEGER, 2001).

Parâmetros capazes de auxiliar na compreensão das relações hídricas em plantas são a eficiência do uso da água (EUA), que nas espécies florestais representa uma relação entre o incremento de biomassa e o volume de água utilizada no período e a evapotranspiração que pode ser definida como a perda de água através da evaporação do solo somada à transpiração das plantas. A evapotranspiração é um processo biofísico que envolve o conteúdo de água do solo, a passagem da água através da planta, a perda de água por transpiração através dos estômatos das folhas e o transporte de água na atmosfera por meio dos processos difusivos e turbulentos (RANA; KATERJI; MASTRORILLI, 1997). Resultados a respeito da evapotranspiração, tanto na produção de mudas quanto em plantios florestais, são de grande importância, pois fornecem informações relativas à quantidade de água consumida pelas plantas, fornecendo dados para o uso eficiente da água nos sistemas de cultivo e dimensionamento de sistemas de irrigação (JUNG et al., 2017).

Em relação aos recursos nutricionais, o nitrogênio é o insumo mais consumido no mundo na agricultura. Esse elemento está presente em inúmeras moléculas orgânicas e está diretamente relacionado ao crescimento e à produtividade das culturas (CRUZ; PAIVA; GUERRERO, 2006). Através da quantificação do nitrogênio total nas mudas, é possível estimar a eficiência com que estas utilizam o nutriente, o que pode fornecer subsídios a produtores e a programas de melhoramento genético visando selecionar espécies capazes de absorver e assimilar o nutriente mesmo sob condições limitantes (CARNEVALI et al., 2016).

Neste contexto, este trabalho buscou comparar o desenvolvimento inicial e a eficiência de uso de água e nitrogênio em mudas de quatro espécies florestais, dentre exóticas e nativas, com potencial para sistemas agroflorestais.

\section{MATERIAL E MÉTODOS}

\section{Material Vegetal}

Foram utilizadas quatro espécies florestais, duas nativas (Calophyllum brasiliense e Tabebuia impetiginosa) e duas exóticas (Eucalyptus urograndis e Toona ciliata), todas com potencial e/ou já sendo utilizadas em florestamentos. As mudas foram obtidas de viveiro de Campo Grande-MS, todas cultivadas em tubetes com substrato orgânico de mesmo volume, apresentando boas condições fitossanitárias e com aproximadamente 120 dias após a semeadura/plantio (DAS). Após remoção do substrato, as mudas foram plantadas em vasos de 8L contendo solo do tipo Latossolo Vermelho-Escuro (EMBRAPA, 1999) previamente corrigido (Tabelas 1 e 2), mantido ao longo do experimento sob $85 \%-90 \%$ da capacidade de campo visando evitar restrição hídrica ou anoxia radicular às mudas. 
TABELA 1: Caracterização granulométrica do solo utilizado no ensaio.

TABLE 1: Granulometric characterization of the soil used in the assay.

\begin{tabular}{cccccc}
\hline & \multicolumn{6}{c}{$\%-\mathrm{g} / \mathrm{kg}$} \\
\hline & & \multicolumn{5}{c}{ Areia } \\
\cline { 2 - 6 } Argila & Silte & Total & Fina & Média & Grossa \\
\hline 68,4 & 16,2 & 15,4 & 10,4 & 4,3 & 0,8 \\
\hline
\end{tabular}

TABELA 2: Caracterização química do solo utilizado no ensaio.

TABLE 2: Chemical characterization of the soil used in the assay.

\begin{tabular}{|c|c|c|c|c|c|c|c|c|c|c|c|c|}
\hline \multicolumn{2}{|c|}{$\mathrm{pH}$} & $\mathrm{P}$ & $\mathrm{K}^{+}$ & $\mathrm{Ca}^{++}$ & $\mathrm{Mg}^{++}$ & $\mathrm{Al}^{3+}$ & $\mathrm{H}^{+}+\mathrm{Al}^{3+}$ & $\mathrm{MO}$ & $\mathrm{S}^{*}$ & $\mathrm{t}$ & $\mathrm{T}$ & $\mathrm{m}$ \\
\hline $\mathrm{H}_{2} \mathrm{O}$ & $\mathrm{CaCl}_{2}$ & \multicolumn{2}{|c|}{$\mathrm{mg} / \mathrm{dm}^{3}$} & \multicolumn{4}{|c|}{$\mathrm{cmol}_{\mathrm{C}} / \mathrm{dm}^{3}$} & $\mathrm{~g} / \mathrm{dm}^{3}$ & \multicolumn{3}{|c|}{$\mathrm{cmol}_{\mathrm{C}} / \mathrm{dm}^{3}$} & $\%$ \\
\hline 5,83 & 4,92 & 0,9 & 32 & 0,3 & 0,3 & 0 & 1,16 & 5,2 & 0,7 & 0,7 & 1,8 & 37 \\
\hline
\end{tabular}

Em que: $\mathrm{S}=$ Soma de Bases; $\mathrm{t}=$ Capacidade de troca Cátions efetiva; $\mathrm{T}=$ Capacidade de Troca Cátions a $\mathrm{pH} 7,0 ; \mathrm{m}$ = Saturação por alumínio; $\mathrm{V}=$ Saturação por bases.

Os vasos foram mantidos sobre mesas em casa de vegetação, recebendo adubações periódicas (100 $\mathrm{mL}$ da solução nutritiva de Hoagland e Arnon (1950) (pH entre 5,6 e 5,9) adicionados à água de reposição de 15 em 15 dias), a fim de garantir plena disponibilidade de nutrientes no solo.

\section{Desenvolvimento inicial e uso de água}

As plantas foram amostradas, destrutivamente, aos 120, 180 e 240 dias após a semeadura. Cada coleta consistiu de oito plantas (vaso/parcela com duas plantas e quatro repetições distribuídas em blocos inteiramente casualizados) por espécie. Logo após, foram separadas em folhas, caule e raiz, tendo suas biomassas frescas determinadas. A área foliar foi mensurada usando um equipamento LI -3100 (Licor, Lincoln, NE, USA) e as raízes lavadas para remoção do solo. A biomassa seca de todas as partes das plantas foi determinada após secagem em estufa de circulação forçada de ar, a uma temperatura de $65^{\circ} \mathrm{C}$ por $72 \mathrm{~h}$ e, posteriormente, pesada em balança de precisão. Após, o material seco foi moído em moinho tipo Wiley para determinação do nitrogênio total (Nt) pelo método de Kjeldahl (SILVA, 2009).

A evapotranspiração foi mensurada diariamente através da pesagem dos vasos em balança de bancada, sendo considerados os valores médios. Vasos sem plantas foram utilizados para estimativa da evaporação da superfície do solo, sendo esses pesados e irrigados com a mesma frequência dos vasos com plantas.

\section{Cálculos}

Com base nos resultados de biomassa seca acumulada e área foliar, foram determinadas, para cada avaliação, a relação raiz/parte aérea, a taxa de crescimento relativo (TCR), a razão de área foliar (RAF), a área foliar específica (AFE), a razão de massa de folha (RMF) e a taxa de assimilação líquida (TAL), de acordo com Hunt et al. (2002). A eficiência de uso de nitrogênio foi estimada com base na porcentagem de nitrogênio total nas mudas.

A eficiência de uso da água (EUA) foi obtida pelo quociente entre o incremento de biomassa (produtividade primária) e o total de água evapotranspirado, de acordo com a equação de Hatfield, Sauer e Prueger (2001). 


\section{Estatística}

As variáveis foram submetidas à análise de variância e subsequente teste de Tukey a 5\% de significância utilizando o programa $\operatorname{Estat}^{\circledR}$ (1994).

\section{RESULTADOS E DISCUSSÃo}

\section{Biomassa Seca, Área Foliar e Relação Raiz/Parte Aérea}

As espécies nativas Calophyllum brasiliense e Tabebuia impetiginosa apresentaram os maiores valores para biomassa seca total (BST) ao fim de 240 DAS, enquanto Toona ciliata apresentou os menores valores para BST no mesmo período (Tabela 3). No entanto, no primeiro período de amostragem, entre 120180 DAS, ficou evidente a variação no acúmulo de biomassa entre espécies exóticas e nativas, em que as espécies nativas apresentaram menor acúmulo em relação à biomassa inicial, acúmulo que foi incrementado no período posterior, enquanto as espécies exóticas, no período de 120-180 DAS, praticamente dobraram sua biomassa total, com redução no período entre 180-240 DAS. Butterfield e Espinoza (1995) avaliando 14 espécies florestais quanto à adaptabilidade na Costa Rica, classificaram Calophyllum brasiliense como uma das espécies florestais mais promissoras pela sua alta sobrevivência e crescimento, forma e volume de madeira, o que corrobora os resultados encontrados neste ensaio, embora Calophyllum brasiliense seja classificada sucessionalmente como climácica, caracterizada pelo crescimento relativamente lento (CARPENTER; NICHOLS; SANDI, 2004). O mesmo pode se afirmar para Tabebuia impetiginosa, classificada como secundária tardia. Contudo, o bom desempenho apresentado quanto ao acúmulo de biomassa pode estar relacionado a outra característica ecológica da espécie, o fato de Tabebuia impetiginosa ser uma espécie decídua (CARVALHO, 1994). Antunez, Retamosa e Villar (2001) avaliando o desenvolvimento inicial de 16 espécies arbóreas relacionadas filogeneticamente e dividindo-as em dois grupos funcionais, decíduas e sempre verdes, observaram que as espécies decíduas cresciam mais rapidamente. Cornelissen, Castro-Díez e Hunt (1996) e Ruiz-Robleto e Villar (2005) encontraram resultados semelhantes.

TABELA 3: Biomassa seca, área foliar e relação raiz/parte aérea de mudas de Calophyllum brasiliense, Eucalyptus urograndis, Toona ciliata e Tabebuia impetiginosa com 120, 180 e 240 dias após a semeadura.

TABLE 3: Dry biomass, leaf area and root shoot ratio of Calophyllum brasiliense, Eucalyptus urograndis, Toona ciliata and Tabebuia impetiginosa seedlings with 120, 180 and 240 days after sowing.

\begin{tabular}{|c|c|c|c|c|c|c|c|c|c|c|c|c|c|}
\hline \multirow[t]{2}{*}{ DAS } & \multirow[t]{2}{*}{ Espécie } & \multicolumn{4}{|c|}{ Biomassa } & \multicolumn{4}{|c|}{ Seca $(g)$} & \multirow[t]{2}{*}{$\begin{array}{l}\text { Área Foliar } \\
\qquad\left(\mathrm{cm}^{2}\right)\end{array}$} & \multicolumn{3}{|c|}{$\begin{array}{l}\text { Relação Raiz/ } \\
\text { Parte Aérea }\end{array}$} \\
\hline & & Folha & & Caule & & Raiz & & Total & & & & & \\
\hline & $\begin{array}{c}\text { Calophyllum } \\
\text { brasiliense }\end{array}$ & 1.51 & $\mathrm{a}$ & 1.95 & a & 1.09 & $\mathrm{~b}$ & 4.56 & $\mathrm{a}$ & 160.83 & $\mathrm{a}$ & 0.33 & $\mathrm{c}$ \\
\hline \multirow[t]{5}{*}{120} & Eucalyptus urograndis & 0.97 & $\mathrm{~b}$ & 0.83 & $\mathrm{~b}$ & 0.93 & $\mathrm{~b}$ & 2.73 & $\mathrm{~b}$ & 105.53 & $\mathrm{~b}$ & 0.52 & $\mathrm{c}$ \\
\hline & Tabebuia impetiginosa & 0.30 & $\mathrm{c}$ & 2.46 & $\mathrm{a}$ & 2.05 & $\mathrm{a}$ & 4.81 & $\mathrm{a}$ & 26.32 & $\mathrm{c}$ & 0.74 & $\mathrm{~b}$ \\
\hline & Toona ciliata & 0.28 & $\mathrm{c}$ & 0.34 & $\mathrm{~b}$ & 0.59 & $\mathrm{~b}$ & 1.2 & $\mathrm{c}$ & 28.09 & $\mathrm{c}$ & 0.95 & $\mathrm{a}$ \\
\hline & CV (\%) & 20.54 & & 23.87 & & 30.37 & & 17.78 & & 10.53 & & 25.41 & \\
\hline & $\begin{array}{c}\text { Calophyllum } \\
\text { brasiliense }\end{array}$ & 2.00 & $\mathrm{a}$ & 2.38 & $\mathrm{a}$ & 2.43 & $\mathrm{a}$ & 6.81 & $\mathrm{a}$ & 177.00 & $\mathrm{~b}$ & 0.56 & $\mathrm{~b}$ \\
\hline \multirow[t]{3}{*}{180} & Eucalyptus urograndis & 1.67 & $a b$ & 1.19 & $\mathrm{~b}$ & 2.17 & $\mathrm{ab}$ & 5.03 & $\mathrm{a}$ & 163.40 & bc & 0.76 & $a b$ \\
\hline & Tabebuia impetiginosa & 1.29 & $\mathrm{~b}$ & 2.21 & $\mathrm{a}$ & 3.01 & $\mathrm{a}$ & 6.51 & $\mathrm{a}$ & 242.13 & $\mathrm{a}$ & 0.86 & a \\
\hline & Toona ciliata & 0.58 & $\mathrm{c}$ & 0.69 & $\mathrm{~b}$ & 1.25 & $\mathrm{~b}$ & 2.52 & $\mathrm{~b}$ & 101.96 & $\mathrm{c}$ & 0.99 & $\mathrm{a}$ \\
\hline
\end{tabular}


TABELA 3: Continuação...

TABLE 3: Continued...

\begin{tabular}{|c|c|c|c|c|c|c|c|c|c|c|c|c|}
\hline \multirow[t]{2}{*}{ DAS } & \multirow[t]{2}{*}{ Espécie } & \multicolumn{4}{|c|}{ Biomassa } & \multicolumn{3}{|c|}{ Seca (g) } & \multirow[t]{2}{*}{$\begin{array}{l}\text { Área Foliar } \\
\left(\mathrm{cm}^{2}\right)\end{array}$} & \multicolumn{3}{|c|}{$\begin{array}{c}\text { Relação Raiz/ } \\
\text { Parte Aérea }\end{array}$} \\
\hline & & Folha & & Caule & & Raiz & & Total & & & & \\
\hline & CV (\%) & 19.44 & & 19.35 & & 21.90 & & 16.59 & 17.42 & & 15.58 & \\
\hline & $\begin{array}{c}\text { Calophyllum } \\
\text { brasiliense }\end{array}$ & 2.69 & $\mathrm{a}$ & 3.53 & $\mathrm{a}$ & 4.2 & $\mathrm{a}$ & $10.42 \mathrm{a}$ & 191.91 & $\mathrm{bc}$ & 0.68 & b \\
\hline \multirow[t]{4}{*}{240} & Eucalyptus urograndis & 2.56 & $\mathrm{a}$ & 2.07 & b & 3.67 & $\mathrm{a}$ & $8.30 \mathrm{~b}$ & 358.38 & $\mathrm{a}$ & 0.79 & b \\
\hline & Tabebuia impetiginosa & 1.38 & $\mathrm{~b}$ & 3.68 & $\mathrm{a}$ & 4.07 & $\mathrm{a}$ & $9.13 \mathrm{ab}$ & 287.03 & $a b$ & 0.80 & b \\
\hline & Toona ciliata & 0.50 & $\mathrm{c}$ & 0.87 & $\mathrm{c}$ & 1.87 & b & $3.23 \mathrm{c}$ & 120.62 & $\mathrm{c}$ & 1.46 & $\mathrm{a}$ \\
\hline & CV (\%) & 20.86 & & 9.26 & & 19.78 & & 12.78 & 19.48 & & 20.40 & \\
\hline
\end{tabular}

Médias seguidas na mesma letra nas colunas não diferem estatisticamente pelo teste de Tukey a 5\% de significância. $\mathrm{CV}=$ coeficiente de variação.

As espécies Tabebuia impetiginosa e Calophyllum brasiliense obtiveram também os maiores rendimentos em biomassa seca de caule (BSC) ao fim de 240 DAS, fato que deve ser levado em consideração já que as diferenças de partição de biomassa para o caule são muito importantes para a tomada de decisões quanto à seleção de materiais genéticos e técnicas de manejo a serem adotadas na condução do povoamento florestal, uma vez que o tronco, de modo geral, é o componente da árvore explorado comercialmente (LADEIRA, 1999), além de contribuir de forma crucial para o pegamento das mudas. Dessa maneira, Tabebuia impetiginosa e Calophyllum brasiliense surgem como potenciais nos meios de produção, uma vez que não houve diferenças estatísticas entre as espécies mencionadas. O desempenho silvicultural de Calophyllum brasiliense vem sendo investigado em vários países da América central, principalmente na Costa Rica onde vem apresentando bons resultados (BUTTERFIELD; ESPINOZA, 1995; JIMÉNEZ et al., 2007). Já existe também um interesse na utilização silvicultural de Tabebuia impetiginosa, porém, é evidente a necessidade de mais estudos a respeito da espécie (SCHNEIDER; SCHNEIDER; FINGER, 2000). O Eucalyptus urograndis com o terceiro maior valor de BSC já tem seu bom desempenho relatado em diversos trabalhos (ODA; MENCK; VENCOVSKY, 1989; FARIA et al., 2008), o que justifica o fato do gênero Eucalyptus spp., representar a base da silvicultura brasileira.

Calophyllum brasiliense e Eucalyptus urograndis apresentaram os maiores incrementos em biomassa seca de folha (BSF) após 240 DAS. Estas espécies apresentaram os maiores valores de BSF ao longo de todo período avaliado. No entanto, no período de 180 DAS, foi observado um marcante investimento em folhas em Tabebuia impetiginosa que não diferiu estatisticamente de Eucalyptus urograndis (Tabela 3), que se deve ao fato desta ser uma espécie caducifólia (CARVALHO, 1994), a qual, no mês do transplante, agosto, apresentava poucas e diminutas folhas.

Já em relação à área foliar (AF), o maior valor obtido a 240 DAS foi em Eucalyptus urograndis o qual não diferiu estatisticamente do valor obtido para Tabebuia impetiginosa. O maior investimento em folhas principalmente em Eucalyptus urograndis e Tabebuia impetiginosa dá a essas espécies vantagens de colonização, pois uma maior área foliar garante uma maior eficiência na interceptação e absorção de radiação fotossinteticamente ativa, o que está diretamente relacionado à produtividade (GRECCO et al., 2011; SILVA et al., 2015). Reis e Barros (1990) afirmam que em Eucalyptus urograndis, até dois anos do plantio, se caracteriza o primeiro estágio de crescimento, no qual há intenso investimento em copa, confirmando os resultados encontrados.

Toona ciliata apresentou a menor biomassa seca de raiz (BSR) após 240 DAS, enquanto as outras espécies avaliadas que apresentaram valores superiores não diferiram entre si estatisticamente para BSR. Já na amostragem inicial de 120 DAS, Tabebuia impetiginosa apresentou o maior valor de BSR, denotando o investimento nesse órgão já nos estágios iniciais de desenvolvimento das mudas. Resultados semelhantes aos apresentados para Tabebuia impetiginosa foram obtidos por Oliveira e Gualtieri (2011) avaliando dife- 
rentes intensidades luminosas no desenvolvimento de Tabebuia aurea, em que, independentemente do nível de sombreamento, as mudas possuíam grande acúmulo de BSR após 120 dias de plantio.

Todas as espécies avaliadas apresentaram alta relação raiz/parte aérea após 240 DAS. No entanto, Toona ciliata se destacou das demais, destinando aproximadamente $58 \%$ da BST na formação de raiz, denotando uma estratégia que visa à maior absorção de água e nutrientes, garantindo maior capacidade para suportar as maiores taxas de fotossíntese e transpiração (CLAUSSEN, 1996). O investimento na formação de raízes pode ser observado também em Calophyllum brasiliense e Eucalyptus urograndis que apresentaram baixa relação raiz/parte aérea após 120 DAS, aumentando nas avaliações seguintes. A espécie nativa Tabebuia impetiginosa apresentou regularidade na relação raiz/parte aérea ao longo do período de avaliação, sendo entre $42 \%$ - 46\% da BST referente à biomassa seca da raiz, o que pode indicar que essa característica seja uma adaptação relacionada a fatores genéticos dessa espécie nas flutuações ambientais às quais são submetidas ao longo de sua evolução no bioma Cerrado, marcado por longos períodos de restrição hídrica, uma vez que os ensaios foram conduzidos visando garantir a disponibilidade hídrica às mudas e minimizar a interferência ambiental relacionada a essa variável. A alta relação raiz/parte aérea juntamente com seu vigoroso desenvolvimento inicial faz a utilização dessa espécie adequada para recomposição vegetal (PEREIRA, 2008).

\section{Variáveis Fisiológicas e Nitrogênio Total}

Quanto às variáveis fisiológicas, a taxa de crescimento relativo (TCR) foi igual estatisticamente para as quatro espécies avaliadas no período entre 120-240 DAS (Figura 1A). Os maiores valores de razão de área foliar (RAF) ao fim de 240 DAS foram obtidos por Eucalyptus urograndis, Toona ciliata e Calophyllum brasiliense que não diferiram estatisticamente (Figura 1B). Uma alta RAF é vantajosa em ambientes de baixa luminosidade, nos quais a interceptação da luz é de primária importância (POORTER, 1999). As espécies Toona ciliata, Tabebuia impetiginosa e Eucalyptus urograndis apresentaram os maiores valores de área foliar específica (AFE) respectivamente, ao fim de 240 DAS, não diferindo estatisticamente (Figura 1C). Especialmente em Eucalyptus urograndis, a alta AFE e RAF melhoram a TCR por conferir às mudas alta interceptação de luz e ganho de carbono por unidade de massa investida em folhas (POORTER; REMKES, 1990; LAMBERS; POORTER, 1992; REICH; WALTERS; ELLSWORTH, 1992; WALTERS; KRUGER; REICH, 1993). No caso de Calophyllum brasiliense, a baixa AFE pode ser resultado da boa disponibilidade de luminosidade na casa de vegetação, tendo em vista que a espécie ocorre naturalmente em bosques e sub-bosques, ambientes em geral sombreados (SEREDA, 2008). 

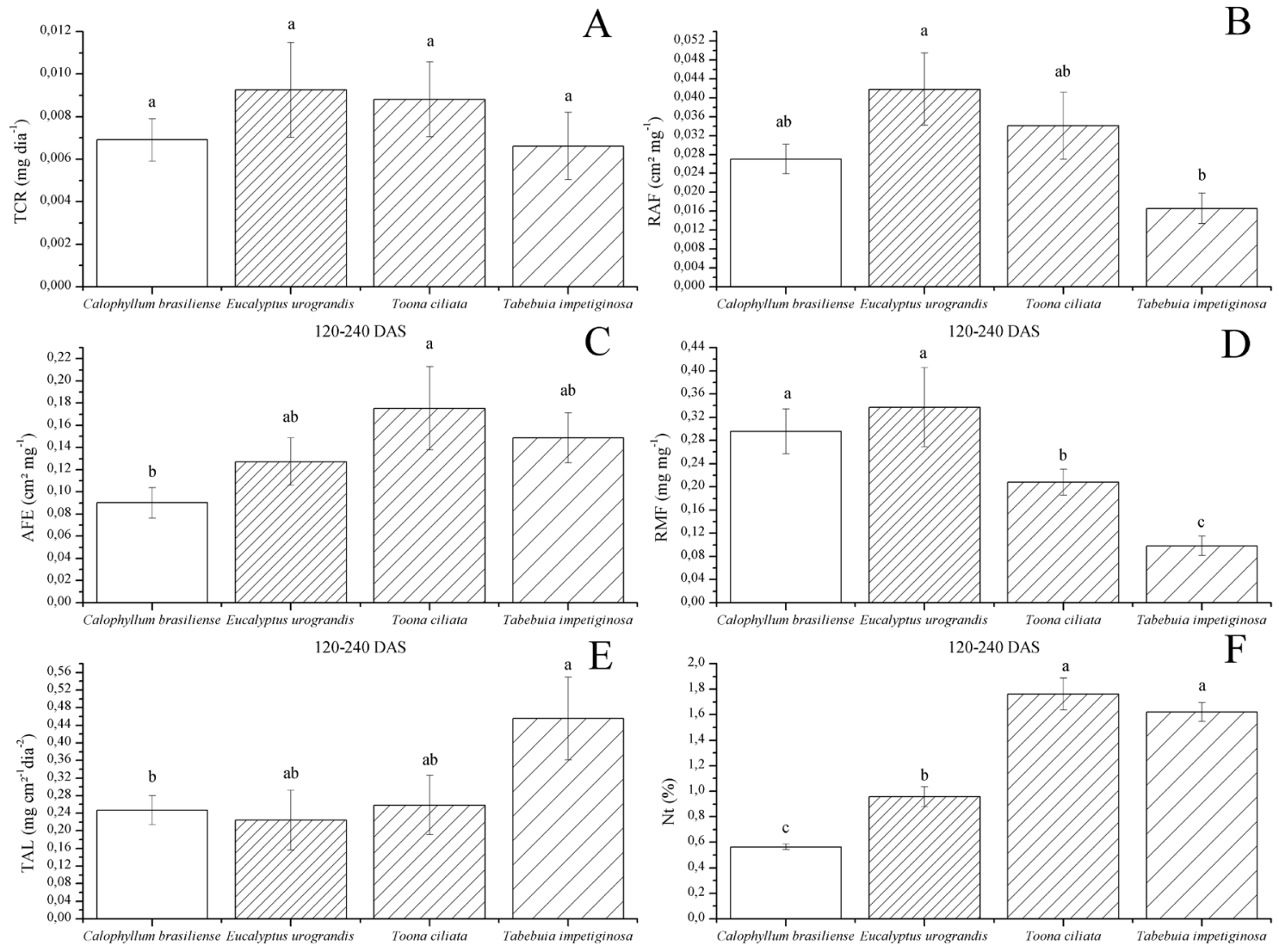

120-240 DAS

120-240 DAS

FIGURA 1: Taxa de crescimento relativo (TCR) (A), razão de área foliar (RAF) (B), área foliar específica (AFE) (C), razão de massa foliar (RMF) (D), taxa de assimilação líquida (TAL) (E), Nitrogênio total (Nt) (F) das espécies Calophyllum brasiliense, Eucalyptus urograndis, Toona ciliata e Tabebuia impetiginosa no período entre 120-240 dias após a semeadura. Barras seguidas da mesma letra não diferem estatisticamente pelo teste de Tukey a $5 \%$ probabilidade.

FIGURE 1: Relative growth rate (RGR) (A), leaf area ratio (LAR) (B), specific leaf area (SLA) (C), leaf mass ratio (LMR) (D), net assimilation rate (NAR) (E), Total Nitrogen (Nt) (F) in Calophyllum brasiliense, Eucalyptus urograndis, Toona ciliata and Tabebuia impetiginosa in the period between 120-240 days after sowing. Bars followed by the same letter do not differ statistically by Tukey test at $5 \%$ probability.

Eucalyptus urograndis e Calophyllum brasiliense apresentam os maiores valores de razão de massa foliar (RMF), com igualdade estatística (Figura 1D). É possível observar que não existe qualquer tendência entre as espécies avaliadas quanto às variáveis AFE e RMF em função de serem exóticas ou nativas, apresentando respostas bem peculiares. Em Eucalyptus urograndis e Calophyllum brasiliense, a RMF é a principal componente na capacidade fotossintética da planta, enquanto em Toona ciliata, há um equilíbrio entre a AFE e a RMF, e em Tabebuia impetiginosa embora também haja certo equilíbrio entre AFE e RMF, a RAF das mudas foi influenciada em maior peso pela AFE. Essa variação de importância entre a AFE e a RMF na RAF e, consequentemente, na TCR é abordada na literatura. Walters, Kruger e Reich (1993) avaliando o desenvolvimento inicial de Betula papyrifera, Betula alleghaniensis, Ostrya virginiana, Quercus rubra e Acer saccharum verificaram que a RMF tem maior importância na TCR. Já Lambers e Poorter (1992), Cornelissen, Castro-Díez e Hunt (1996) e Ruiz-Robleto e Villar (2005), avaliando diversas espécies florestais, verificaram que a AFE possui maior importância na TCR. Claussen (1996) afirma que uma alta RMF e baixa AFE (folhas mais espessas) seriam benéficas ao passo que menos material vegetal por unidade de área seria diretamente exposto à luz, reduzindo a perda de água e aumentando o autossombreamento entre cloroplastos. O mesmo autor afirma ainda que uma baixa RMF (folhas mais delgadas) poderia trazer vantagens às plantas expostas à elevada radiação fotossinteticamente ativa como maior investimento em 
biomassa de caule e raiz, tendo como consequência a ampliação de sua capacidade de absorção de nutrientes. Esse último panorama foi encontrado em Toona ciliata e Tabebuia impetiginosa, e pode ser reforçado com base nos dados de biomassa seca e relação raiz/parte aérea. Especialmente no caso de Tabebuia impetiginosa, sendo uma espécie decídua, possui de folhas de vida curta não-escleromorfas que são caracterizadas pela alta capacidade fotossintética e grande atividade do metabolismo do nitrogênio (LARCHER, 2006), o que pôde ser observado através da taxa de assimilação líquida (TAL) e da quantificação de nitrogênio total (Figuras 1E e F). Tabebuia impetiginosa seguida de Toona ciliata e Eucalyptus urograndis apresentaram os maiores valores de TAL, não diferindo estatisticamente (Figura 1E). A TAL representa o incremento de material vegetal por unidade de área foliar e de tempo, ou seja, a taxa de fotossíntese líquida. Esta característica de crescimento sofre menor influência da ontogenia da planta do que a TCR e é dependente da radiação solar, das condições internas da planta, do próprio índice de área foliar e do balanço hídrico (CONCEIÇÃO; LOPES; FORTES, 2005). Tendo em vista que o ensaio foi realizado em condições controladas, os valores da TAL expressam as diferenças inerentes a cada espécie quanto à assimilação de carbono. Em todas as espécies avaliadas a TAL deu a maior contribuição para a TCR. Essa constatação significa que grande parte da TCR pode ser explicada pela eficiência fotossintética da espécie (POORTER, 1999). Resultados semelhantes foram encontrados por Crisóstomo, Freitas e Rodríguez-Echeverría (2007) em trabalho realizado com Acacia longifolia, Cytisus grandiflorus e Ulex europaeus, no qual a TAL demonstrou maior importância na TCR. Poorter (1999) avaliando 15 espécies florestais também verificou que a TAL tem maior importância na TCR de diferentes espécies, e ainda acrescenta que essa condição é mais bem observada em condições de alta luminosidade. Contudo, Antunez, Retamosa e Villar (2001) verificaram que as variações na TCR entre espécies florestais são atribuídas em sua maioria à RAF, tendo a AFE como principal componente.

Toona ciliata e Tabebuia impetiginosa apresentaram os maiores valores de nitrogênio total, sendo significativamente superiores a Eucalyptus urograndis e Calophyllum brasiliense, respectivamente (Figura $1 F)$. Esses resultados reforçam a relação entre altos valores de AFE com a maior eficiência na assimilação de nutrientes. O nitrogênio é considerado elemento essencial para as plantas, pois está presente na composição das mais importantes biomoléculas, tais como ATP, NADH, NADPH, clorofila, proteínas e inúmeras enzimas. Tendo em vista que menos de $50 \%$ do nitrogênio aplicado sob a forma de fertilizantes é aproveitado, a utilização de espécies mais eficientes na assimilação de nitrogênio é algo desejável (BREDEMEIER; MUNDSTOCK, 2000). A maior eficiência na assimilação de nitrogênio está estreitamente relacionada a aumentos de produtividade, pois esse elemento atua favorecendo o aumento da fotossíntese através de sua participação na síntese de clorofilas e das enzimas fosfoenolpiruvato carboxilase (PEPC) e ribulose 1,5 bifosfato carboxilase/oxigenase (Rubisco), que participam da fixação de $\mathrm{CO}_{2}$ atmosférico (PRADO, 2008). Os resultados encontrados corroboram os apresentados por Poorter e Remkes (1990) e Poorter, Remkes e Lambers (1990) avaliando a RAF, TAL, economia de carbono e nitrogênio em 24 espécies herbáceas de metabolismo $\mathrm{C}_{3}$, em que maiores níveis de nitrogênio favoreceram maiores taxas fotossintéticas.

\section{Evapotranspiração e Eficiência de Uso de Água (EUA)}

Eucalyptus urograndis apresentou a maior evapotranspiração ao longo de todo período de avaliação, diferindo estatisticamente das demais e seguida pelas espécies Calophyllum brasiliense e Tabebuia impetiginosa (Tabela 4). Nota-se que não há uma distinção entre as espécies exóticas e nativas quanto à evapotranspiração, sendo que a espécie que apresentou os menores valores ao longo do ensaio foi Toona ciliata, espécie exótica. No entanto, com base nos resultados, é possível distinguir as espécies em grupos funcionais, tendo em vista que Toona ciliata também se trata de uma espécie decídua (RICKEN et al., 2011), o que parece fazer mais sentido. Toona ciliata e Tabebuia impetiginosa (decíduas), que apresentaram os menores valores de evapotranspiração, dispõem de mecanismos avançados de economia de água que vão desde a abscisão das folhas (POPMA; BONGERS; WERGER, 1992) até alta eficiência na assimilação de nitrogênio (LARCHER, 2006). A menor evapotranspiração apresentada por esses materiais passa a fazer ainda mais sentido quando se toma por base os parâmetros fisiológicos, em especial a AFE. Os maiores valores de AFE induzem a uma maior TAL e elevação da assimilação de nitrogênio e dos níveis citoplasmáticos desse elemento, o que atua diretamente no controle estomático, reduzindo a condutância e a perda 
de água através da transpiração, o que também favorece o aumento da TAL (MEZIANE; SHIPLEY, 2001; QUERO et al., 2006).

TABELA 4: Evapotranspiração por parcela e eficiência de uso de água das mudas (EUA) de Calophyllum brasiliense, Eucalyptus urograndis, Toona ciliata e Tabebuia impetiginosa nos períodos entre 120-180, 180-240 e 120-240 (Total) dias após a semeadura.

TABLE 4: Evapotranspiration per plot and water use efficiency of seedlings Calophyllum brasiliense, Eucalyptus urograndis, Toona ciliata and Tabebuia impetiginosa in the periods between 120-180, 180-240 and 120-240 (Total) days after sowing.

\begin{tabular}{|c|c|c|c|c|c|c|c|c|}
\hline \multirow{2}{*}{ Espécie } & \multicolumn{5}{|c|}{ Evapotranspiração $\left(\mathrm{g} \mathrm{H}_{2} \mathrm{O}\right)$ por parcela ${ }^{1}$} & \multirow{2}{*}{\multicolumn{3}{|c|}{$\frac{\text { EUA }}{\mathrm{mg} \mathrm{g}^{-1} \mathrm{H}_{2} \mathrm{O}}$}} \\
\hline & \multicolumn{2}{|c|}{ 120-180 DAS } & \multicolumn{2}{|c|}{ 180-240 DAS } & Total & & & \\
\hline Calophyllum brasiliense & 10142.50 & $\mathrm{~b}$ & 10823.75 & b & 20966.25 & $\mathrm{~b}$ & 0.7758 & a \\
\hline Eucalyptus urograndis & 11265.00 & $\mathrm{a}$ & 12503.75 & $\mathrm{a}$ & 23768.75 & $\mathrm{a}$ & 0.5825 & $\mathrm{~b}$ \\
\hline Tabebuia impetiginosa & 9755.00 & $\mathrm{~b}$ & 8598.75 & $\mathrm{c}$ & 18353.75 & $\mathrm{c}$ & 0.7321 & $a b$ \\
\hline Toona ciliata & 8687.50 & $\mathrm{c}$ & 8263.75 & $\mathrm{c}$ & 16951.25 & $\mathrm{~d}$ & 0.3095 & c \\
\hline Controle (Solo) & 8556.25 & $\mathrm{c}$ & 8083.75 & $\mathrm{c}$ & 16640.00 & $\mathrm{~d}$ & -------. & \\
\hline $\mathrm{CV}(\%)$ & 4.34 & & 3.38 & & 3.23 & & 15.32 & \\
\hline
\end{tabular}

${ }^{1}$ Cada parcela é composta por duas plantas. Médias seguidas na mesma letra nas colunas não diferem estatisticamente pelo teste de Tukey a 5\% de significância. CV = coeficiente de variação.

Calophyllum brasiliense e Tabebuia impetiginosa apresentaram as maiores EUA seguidas por Eucalyptus urograndis (Tabela 4). A alta EUA apresentada por Calophyllum brasiliense pode ser justificada pelo fato de a espécie possuir folhas esclerófilas, mais espessas e tolerantes ao estresse hídrico (EDWARDS; READ; SANSON, 2000; SEREDA, 2008). Em Tabebuia impetiginosa, como anteriormente mencionado, um rigoroso controle estomático aliado ao desenvolvimento e abscisão foliar peculiar às espécies decíduas parece suficiente para justificar a alta EUA observada. Essas características são adaptações dessas espécies às variações ambientais. A espécie é frequente no cerradão, cerrado, caatinga e mata seca (CARVALHO, 1994), regiões em que, frequentemente, as espécies são submetidas a restrições hídricas durante parte do ano.

Já em Eucalyptus urograndis, a alta área foliar, e as intensas ramificações da parte aérea observadas para espécie, podem ser apresentadas como razões para a EUA apresentada. Segundo Ogbonnaya et al. (1998) e Bañon et al. (2004), as ramificações geram maior superfície, o que consequentemente gera maior utilização de água do solo.

Devido destaque deve ser dado à menor EUA em Toona ciliata que mesmo sendo uma espécie decídua e obtendo menores valores de evapotranspiração, foi a espécie menos eficiente pelo seu mínimo incremento em biomassa ao longo do ensaio. Esse baixo acúmulo de biomassa relatado vai na contramão da literatura que se refere à espécie, na qual é frequentemente descrita como de rápido crescimento (RICKEN et al., 2011). No entanto, justificativas para esse baixo desempenho podem residir no fato de que a espécie não se desenvolve bem em ambientes permanentemente encharcados e solos argilosos compactados, reduzindo sua taxa de crescimento (SOUZA; BARROSO; CARNEIRO, 2010). Embora, no ensaio, a espécie não tenha sido mantida em ambiente encharcado, a umidade do solo mantida foi bastante alta $(85 \%-90 \%$ da capacidade de campo) por longo período. Adicionalmente, o solo argiloso utilizado possui grande capacidade de retenção de água, tornando-se pesado, o que pode ter prejudicado o desenvolvimento da espécie.

\section{CONCLUSÕES}

Calophyllum brasiliense,Tabebuia impetiginosa e Eucalyptus urograndis apresentaram os melhores desempenhos no acúmulo de biomassa seca e nas variáveis fisiológicas auferidas no período entre 
120-240 DAS. As duas primeiras espécies surgem como alternativas promissoras nos florestamentos com espécies nativas, carecendo de mais estudos.

Eucalyptus urograndis apresentou a maior evapotranspiração, porém, com bom acúmulo de massa e Calophyllum brasiliense e Tabebuia impetiginosa foram as espécies mais eficientes no uso de água, enquanto Tabebuia impetiginosa e Toona ciliata foram as espécies mais eficientes no uso de nitrogênio.

\section{AGRADECIMENTOS}

A FUNDECT pelo auxílio financeiro e concessão de bolsa, a Embrapa Gado de Corte pela cessão da casa de vegetação e laboratórios, a Alex Marcel Melotto pela doação de mudas e a Isaura Megumi Naka, José Porfírio da Silva e Marcelo Leandro Bueno pelas contribuições na execução do trabalho.

\section{REFERÊNCIAS}

AGUIAR-NETTO, A. O.; RODRIGUES, J. D.; PINHO, S. Z. Análise de crescimento na cultura da batata submetida a diferentes lâminas de irrigação. Pesquisa Agropecuária Brasileira, Brasília, v. 35, p. 901-907, 2000.

ALVAREZ, R. C. F. et al. Análise de crescimento de duas cultivares de amendoim (Arachis hypogaea L.). Acta Scientiarum Agronomy, Maringá, v. 27, n. 4, p. 611-616, 2005.

ANTUNEZ, I.; RETAMOSA, E. C.; VILLAR, R. Relative growth rate in phylogenetically related deciduous and evergreen woody species. Oecologia, Berlin, v. 128, p. 172-180, 2001.

BAÑON, S. et al. Effects of water stress and night temperature preconditioning on water relations and morphological and anatomical changes of Lotus creticus plants. Scientia Horticulturae, Amsterdam, v. 101, p. 333-342, 2004.

BENINCASA, M. M. P. Análise de crescimento de plantas, noções básicas. Jaboticabal: FUNEP, 2003. v. $2.42 \mathrm{p}$.

BREDEMEIER, C.; MUNDSTOCK, C. M. Regulação da absorção e assimilação do nitrogênio nas plantas. Ciência Rural, Santa Maria, v. 30, n. 2, p. 365-372, 2000.

BUTTERFIELD, R.; ESPINOZA, M. Screening trial of 14 tropical hardwoods with an emphasis on species native to Costa Rica: fourth year results. New Forests, Dordrecht, v. 9, p. 135-145, 1995.

CARNEVALI, N. H. S. et al. Eficiência nutricional de mudas de Stryphnodendron polyphyllum em função de nitrogênio e fósforo. Ciência Florestal, Santa Maria, v. 26, n. 2, p. 449-461, 2016. http://dx.doi. org/10.5902/1980509822746.

CARPENTER, F. L.; NICHOLS, J. D.; SANDI, E. Early growth of native and exotic trees planted on degraded tropical pasture. Forest Ecology and Management, Amsterdam, v. 196, p. 367-378, 2004.

CARVALHO, P. E. R. Espécies Florestais Brasileiras. Recomendações Silviculturais, potencialidades e uso da madeira. Brasília: EMBRAPA; CNPF, 1994. 640 p.

CLAUSSEN, J. W. Acclimation abilities of three tropical rainforest seedlings to an increase in light intensity. Forest Ecology and Management, Amsterdam, v. 80, p. 245-255, 1996.

CONCEIÇÃO, M.; LOPES, N.; FORTES, G. Análise de crescimento de plantas de batata-doce (Ipomoea batatas L.) cultivares abóbora e da costa. Revista Brasileira de Agrociência, Pelotas, v. 11, n. 3, p. 273-278, 2005.

CORNELISSEN, J. H. C.; CASTRO-DÍEZ, P.; HUNT, R. Seedling growth, allocation and leaf attributes in a wide range of woody plant species and types. Journal of Ecology, London, v. 84, p. 755-765, 1996.

CRISÓSTOMO, J. A.; FREITAS, H.; RODRÍGUEZ-ECHEVERRÍA, S. Relative growth rates of three woody legumes: implications in the process of ecological invasion. Web Ecology, [s. 1.], v. 7, p. 22-26, 2007.

CRUZ, C. A. F. et al. Efeito de diferentes níveis de saturação por bases no desenvolvimento e qualidade de mudas de ipê-roxo (Tabebuia impetiginosa (Mart.) Standley). Scientia Forestalis, Piracicaba, v. 66, p. 100-107, 2004.

CRUZ, C. A. F.; PAIVA, H. N.; GUERRERO, C. R. A. Efeito da adubação nitrogenada na produção de 
mudas de sete-cascas (Samanea inopinata (Harms) Ducke). Revista Árvore, Viçosa, MG, v. 30, n. 4, p. 537-546, 2006.

DOORENBOS, J.; KASSAN, A. H. Yield response to water. Rome: FAO, 1979. 193 p. (Irrigation and Drainage Paper, 33).

EDWARDS, C.; READ, J.; SANSON, G. Characterisingsclerophylly: some mechanical propertis of leaves from heath and forest. Oecologia, Berlin, v. 123, p. 158-167, 2000.

EMBRAPA. Sistema brasileiro de classificação de solos. Brasília: EMBRAPA, 1999. 412 p.

ESTAT. Sistema de análises estatísticas. Jaboticabal: FCAV; UNESP, 1994.

FARIA, G. E. et al. Avaliação da produtividade, conteúdo e eficiência de utilização de nutrientes em genótipos de Eucalyptus spp. no Vale do Jequitinhonha, MG. Ciência Florestal, Santa Maria, v. 18, n. 3, p. 363-373, 2008.

FINGER, C. A. G. et al. Estabelecimento de povoamentos de Pinus elliottii Engelm pela semeadura direta a campo. Ciência Florestal, Santa Maria, v. 13, n. 1, p. 107-113, 2003.

GONÇALVES, J. L. M. et al. Produção de mudas de espécies nativas: substrato, nutrição, sombreamento e fertilização. In: GONÇALVES, J. L. M.; BENEDETTI, V. (Ed.). Nutrição e fertilização florestal. Piracicaba: IPEF, 2000. p. 309-350.

GRAVE. F. et al. Crescimento de plantas jovens de açoita-cavalo (Luehea divaricata) em quatro diferentes substratos. Ciência Florestal, Santa Maria, v. 17, n. 4, p. 289-298, 2007.

GRECCO, E. D. et al. Estimativa do índice de área foliar e determinação do coeficiente de extinção luminosa da abóbora Cucurbita moschata var. japonesa. Idesia, Arica, v. 29, n. 1, p. 37-41, 2011. https://dx.doi.org/10.4067/S0718-34292011000100006

GROOM, P. K.; LAMONT, B. B. Which common indices of sclerophylly best reflect differences in leaf structure? Ecoscience, Saint-Foy, v. 6, n. 3, p. 471-474, 1999.

HATIFIELD, J. L.; SAUER, T. J.; PRUEGER, J. H. Managing soils to achieve greater water use efficiency: a review. Agronomy Journal, Madison, v. 93, p. 271-280, 2001.

HOAGLAND, D. R.; ARNON, D. I. The water culture method for growing plants without soils. Berkeley: California Agricultural Experimental Station, 1950. 347 p.

HUNT, R. et al. A modern tool for classical plant growth analysis. Annals of Botany, Oxford, v. 90, p. 485-488, 2002.

JIMÉNEZ, J. J. et al. Soil organic carbon pool under native tree plantations in the Caribbean lowlands of Costa Rica. Forest Ecology and Management, Amsterdam, v. 241, p. 134-144, 2007.

JUNG, L. H. et al. Irrigation in initial development of Eucalyptus urophylla x Eucalyptus grandis and Eucalyptus grandis x Eucalyptus camaldulensis. Ciência Florestal, Santa Maria, v. 27, n. 2, p. 655-667, 2017. http://dx.doi.org/10.5902/1980509827750.

LADEIRA, B. C. Crescimento, produção de biomassa e eficiência nutricional de Eucalyptus spp., sob três espaçamentos, em uma seqüência de idades. 1999. 132 f. Dissertação (Mestrado em Ciência Florestal) - Universidade Federal de Viçosa, Viçosa, MG, 1999.

LAMBERS, H.; POORTER, H. Inherent variation in growth rate between higher plants: a search for physiological causes and ecological consequences. Advances in Ecological Research, London, v. 23, p. 187-261, 1992.

LARCHER, W. Ecofisiologia Vegetal. São Carlos: Rima, 2006. 550 p.

LORENZI, H. et al. Árvores Exóticas no Brasil: madeireiras, ornamentais e aromáticas. Nova Odessa: Plantarum, 2003. $385 \mathrm{p}$.

MARIMON, B. S. et al. Desenvolvimento inicial e partição de biomassa de Brosimum rubescensTaub. (Moraceae) sob diferentes níveis de sombreamento. Acta Botanica Brasilica, Belo Horizonte, v. 22, n. 4, p. 941-953, 2008.

MEZIANE, D.; SHIPLEY, B. Direct and indirect relation between specific leaf area, leaf nitrogen and leaf gas exchange effects of irradiance and nutrient supply. Annals of Botany, Oxford, v. 88, p. 915-917, 2001. MOTA, L. H. S.; SCALON, S. P. Q.; HEINZ, R. Sombreamento na emergência de plântulas e no crescimento inicial de Dipteryx alata Vog. Ciência Florestal, Santa Maria, v. 22, n. 3, p. 423-431, 2012.

ODA, S; MENCK, A. L. M.; VENCOVSKY, R. Problemas no melhoramento genético clássico do eucalipto em função da alta intensidade de seleção. Instituto de Pesquisas e Estudos Florestais, Piracicaba, 
v. 41/42, n. 3 , p. $8-17,1989$.

OGBONNAYA, C. I. et al. Growth and water relations of Kenaf (Hibiscus cannabinus L.) under water deficit on a sandy soil. Ind. Crop Production, Washington, v. 8, p .65-76, 1998.

OLIVEIRA, A. D. et al. Avaliação econômica da vegetação de cerrado submetida a diferentes regimes de manejo e de povoamentos de eucalipto plantado em monocultivo. Cerne, Lavras, v. 4, n. 1, p. 34-56, 1998. OLIVEIRA, A. K. M.; GUALTIERI, S. C. J. Crescimento inicial de Tabebuia aurea sob três intensidades luminosas. REVSBAU, Piracicaba, v. 6, n. 2, p. 90-103, 2011.

PEREIRA, A. R. Como selecionar plantas para áreas degradadas e controle de erosão. 2. ed. Belo Horizonte: FAPI, 2008. 239 p.

POORTER, H.; REMKES, C. Leaf area ratio and net assimilation rate of 24 wild species differing in relative growth rate. Oecologia, Berlin, v. 83, p. 553-559, 1990.

POORTER, H.; REMKES, C.; LAMBERS, H. Carbon and nitrogen economy of 24 wild species differing in relative growth rate. Plant Physiology, Bethesda, v. 94, p. 621-627, 1990.

POORTER, L. Growth responses of 15 rain-forest tree species to a light gradient: the relative importance of morphological and physiological traits. Functional Ecology, London, v. 13, p. 396-410, 1999.

POPMA, J.; BONGERS, F.; WERGER, M. J. A. Gap-dependence and leaf characteristics of trees in a tropical lowland rain forest in Mexico. Oikos, Copenhagen, v. 63, p. 207-214, 1992.

PRADO, R. M. Nitrogênio. In: PRADO, R. M. (Ed.). Nutrição mineral de plantas. São Paulo: Editora UNESP, 2008. $407 \mathrm{p}$.

QUERO, J. L. et al. Interactions of drought and shade effects on seedlings of four Quercus species: physiological and structural leaf responses. New Phytologist, Cambridge, v. 170, p. 819-834, 2006.

RANA, G.; KATERJI, N.; MASTRORILLI, M. Environmental and soil-plant parameters for modeling actual crop evapotranspiration under water stress conditions. Ecological Modelling, Amsterdam, v. 101, p. 363-371, 1997.

REICH, P. B.; WALTERS, M. B.; ELLSWORTH, D. S. Leaf lifespan in relation to leaf plant and stand characteristics among diverse ecosystems. Ecological Monographs, Lawrence, v. 62, p. 365-392, 1992.

REIS, M. G. F.; BARROS, N. F. Ciclagem de nutrientes em plantios de eucalipto. In: BARROS, N. F.; NOVAIS, R. F. (Ed.). Relação solo-eucalipto. Viçosa, MG: Ed. Folha de Viçosa, 1990. p. 265-296.

RICKEN, $\mathrm{P}$. et al. Crescimento diamétrico de povoamento de Toona ciliata var. australis em Adrianópolis, PR. Colombo: EMBRAPA, 2011. (Comunicado Técnico, n. 285).

RUIZ-ROBLETO, J.; VILLAR, R. Relative growth rate and biomass allocation in 10 woody species with different leaf longevity using phylogenetic independent contrasts PICs. Plant Biology, Stuttgart, v. 7, p. 484-494, 2005.

SILVA, S. F. et al. Modelos alométricos para estimativa da área foliar de mangueira pelo método não destrutivo. Revista Agro@mbiente On-line, Boa Vista, v. 9, n. 1, p. 86-90, 2015.

SCHNEIDER, P. S. P.; SCHNEIDER, P. R.; FINGER, C. A. G. Crescimento do ipê-roxo, Tabebuia impetiginosa Martius ex A. P. de Candolle, na depressão central do estado do rio grande do sul. Ciência Florestal, Santa Maria, v. 10, n. 2, p. 91-100, 2000.

SEREDA, F. Caracterização nutricional e grau de esclerofilia foliar de guanandi em floresta ombrófila densa no litoral do Paraná. 2008. 80 f. Dissertação (Mestrado) - Universidade Federal do Paraná, Curitiba, 2008.

SILVA, F. C. Manual de análises químicas de solos, plantas e fertilizantes. 2. ed. rev. ampl. Brasília: EMBRAPA; SCT, 2009. 627 p.

SILVA, L. C.; BELTRÃO, N. E. M.; AMORIM NETO, M. S. Análise do crescimento de comunidades vegetais. Campina Grande: EMBRAPA; CNPA, 2000. 18 p. (Circular Técnica, 34).

SOCIEDADE BRASILEIRA DE SILVICULTURA. Fatos e Números do Brasil Florestal. [2008]. Disponível em: <http://www.sbs.org.br/FatoseNumerosdoBrasilFlorestal.pdf > Acesso em: 27 fev. 2013.

SOUZA, J. C. A. V.; BARROSO, D. G.; CARNEIRO, J. G. A. Cedro Australiano (Toona ciliata). Niterói: Programa Rio Rural, 2010. 12 p. (Manual Técnico, 21).

WALTERS, M. B.; KRUGER, E. L.; REICH, P. B. Relative growth rate in relation to physiological and morphological traits for northern hardwood seedlings: species, light environment and ontogenetic considerations. Oecologia, Berlin, v. 96, p. 219-231, 1993. 